\title{
ORIENTACION DE LA ENFERMERA PARA EL CUIDADO EN EL HOGAR DEL PACIENTE CON ALZHEIMER
}

Edith Johanna Hernández Garzón, Ana Lucia Hormiga Marín, Laura Ximena Huérfano Niño, Solanyi Leguizamón Figueredo, Gladys Esperanza Rozo Riveros.*

\section{RESUMEN}

En los últimos años vemos como la enfermedad de Alzheimer, va tomando relevancia en cuanto a número de muertes se refiere, ya que en sí es una enfermedad degenerativa, progresiva y con alta incapacidad que acaba irremediablemente en la muerte. Al ser ésta una enfermedad de larga evolución que va mermando en las personas tanto sus capacidades físicas como cognitivas, el paciente va a ser cada vez más dependiente de los cuidados de sus familiares; así es como aparece la figura del cuidador principal. Se contó con la participación de once familias de diferentes áreas de Bogotá y pertenecientes a los estratos entre 2 y 4 , que tienen en su hogar un integrante con este problema, con el fin de detectar conceptos, conocimientos y creencias que poseían en relación con esta patología y de esta forma implementar una estrategia eficaz para la orientación en el cuidado de este grupo de pacientes utilizando un tipo de estudio cuantitativo cuasiexperimental y la prueba estadística de Mc Nemar para la validación de la hipótesis. Se estructuró un pretest para identificar el déficit de conocimientos en las áreas de: medio ambiente, recreación, sentimientos, sexualidad, nutrición y manejo de higiene, úlceras, curaciones e incontinencia; con base en la información obtenida, se realizaron tres sesiones educativas personalizadas, buscando que las familias vinculadas al estudio tuvieran una comprensión de los cambios que se presentan durante el curso de la enfermedad y, de esta manera, entendieran la importancia del cuidado integral que requiere este tipo de pacientes en el hogar. Posteriormente se aplicó el postest donde se observó que el nivel de conocimientos adquirido por las familias permitió que se cumpliera el propósito de la investigación que consistió en orientar a la familia para que identificara los cuidados integrales en el hogar que debe tener un paciente con enfermedad de Alzheimer, para así crear un ambiente de convivencia donde haya calidad de vida en el entorno familiar.

\section{Introducción}

A medida que la población mundial va creciendo, su esperanza de vida aumenta y, por lo tanto, las personas mueren más ancianas y son susceptibles a padecer enfermedades degenerativas propias de la edad. Entre las más relevantes están las demencias, en especial el Alzheimer debido a la gran cantidad de casos que se presentan y al proceso degenerativo al que lleva al paciente.

La prevalencia es de $5 \%$ en personas mayores de 65 años y de 30-40\% en estadios leves. Además, es la tercera causa de muerte después de las afecciones cardiovasculares y el cáncer. ${ }^{1}$

Estudiantes de VII Semestre de Enfermería de la Fundación Universitaria de Ciencias de la Salud, Bogotá.
La enfermedad de Alzheimer (EA) es una demencia degenerativa primaria de causa casi desconocida con una máxima frecuencia esporádica aunque se sabe que puede ser familiar o hereditaria en el 5-10\% de los casos.

Los datos estadísticos muestran un importante crecimiento permanente de los pacientes diagnosticados con este tipo de enfermedad, como se estableció en la III Reunión Latinoamericana de Alzheimer, realizada en la Habana, Cuba, los días 28-31 de marzo de 2000, donde se concluyó que cada vez se presenta en el mundo un mayor número de casos con algún tipo de demencia senil; el más relevante es la enfermedad de Alzheimer. ${ }^{2}$

Según la Asociación interdisciplinaria de Gerontología de Colombia, hacia el año 2025 las personas mayores de 55 años sumarán más de 11.000.200 (aproximadamente $19 \%$ del conjunto de la población) y dentro de 50 
años, su volumen será alrededor de 20.000.000, lo que representará la cuarta parte del total de los colombianos. ${ }^{3}$

Con el gran avance tecnológico (técnicas inmunohistoquímicas y microscopía electrónica) se han logrado describir diferentes tipos de lesiones, por medio de tinciones argénticas, hematoxilina y eosina y métodos para la determinación de amiloide. Estas lesiones clásicas son: Ovillos neurofibrilares (se presentan como masas de fibrilla entrelazadas, acumuladas en el cuerpo neural y con los métodos se tiñen de color plata, se ubican especialmente en el citoplasma perinuclear y toman una figura de «llama» en las neuronas corticales y de «ovillo» en las subcorticales); placas seniles (se encuentra en ancianos sanos; aparecen como zonas circulares de 100 nm con dos componentes básicos: un núcleo central de amiloide extendido hacia la periferia y una corona de neuritas anormales, entre las cuales se encuentran células microgliales; por medios bioquímicos se han demostrado las características del amiloide al ser diferenciado de otras sustancias que se encuentran fuera del sistema nervioso central); cuerpos de Hirano (formaciones ovoideas, altamente eosinofílicas, que recubren los somas neuronales; en el microscopio electrónico se observan como láminas fibrilares paralelas compuestas por actina y varias proteínas ligadas a ellas, como la tropomiosina); angiopatía congofilica (acúmulo de sustancias amiloides en la capa media de las arterias y arteriolas de la corteza cerebral y las meninges, su nombre se debe a la identificación de la coloración rojo congo); pérdida neuronal (debida a la acumulación de la sustancia amiloide en el citoplasma de la célula, que lleva a la degeneración y posteriormente a su muerte) ${ }^{4}$

La pérdida neuronal se produce en áreas de asociación de las cortezas frontal, temporal y parietal de ambos hemisferios, por lo cual quedan indemnes las cortezas motora primaria, somatosensitiva, visual y auditiva. Además de la pérdida neuronal, los signos histopatológicos más característicos son las placas seniles (neuríticas) de material amorfo (amiloide), engrosamiento y condensación del componente neurofibrilar de las neuronas. ${ }^{5}$

El diagnóstico más confiable de esta enfermedad se realiza por medio del examen anatomopatológico de una muestra de sustancia cerebral. No obstante, existe una serie de criterios diagnósticos sin que el paciente haya fallecido.

La orientación que debe recibir la familia de estos pacientes con EA está fundamentada en el cuidado integral que requieren en el hogar, teniendo en cuenta que son seres biosicosociales que necesitan un ambiente adecuado para desenvolverse e interactuar con otros de la forma menos traumática y dolorosa posible, pues, pese a las limitaciones propias de su enfermedad, siguen perteneciendo a un contexto social cuya célula fundamental es la familia.

La familia debe construir, basada en el afecto, un compromiso que la llevará a desempeñar adecuadamente el papel de cuidador y guiador en el transcurso de la vida cotidiana del paciente. Se sabe que las personas con problemas de comportamiento, entre las que se encuentran los pacientes de este grupo, necesitan un apoyo físico y emocional en cada una de las etapas de la enfermedad, puesto que conlleva un proceso de pérdida de funciones y cambios conductuales, siendo las más significativas la pérdida de la memoria, la desorientación, la hipoprosexia, las alucinaciones, los delirios, la agnosia, las alteraciones del sueño, la apraxia y los trastornos de las funciones cerebrales en general

La EA se divide en tres etapas, según su evolución; por lo tanto, el profesional de enfermería desempeña uno de los papeles más importantes en la orientación respecto al cuidado y manejo de este tipo de patología, ya que el objetivo de la profesión es velar porque se brinde una atención de calidad a todas las personas y comunidades, sin distinción de clases socioeconómicas, etnias, edad, sexo, religión, área geográfica u otra condición. ${ }^{6}$

\section{Materiales y métodos}

En esta investigación se utilizó un método cuantitativo seleccionando una población de once familias de pacientes con EA que tuvieran a su cargo el cuidado directo de los mismos, que de manera voluntaria participaran en el proyecto, ubicadas en diferentes áreas de Bogotá y dentro del periodo establecido para el estudio (segundo semestre de 2001-segundo semestre de 2002), utilizando un tipo de muestreo no probabilístico. 
Para la recolección de datos, se utilizó un pretest, el cual se diseñó de tal forma que se pudieran detectar los conocimientos de la familia sobre los aspectos relacionados con el cuidado brindado al paciente en el hogar. Este contenía 14 preguntas de selección múltiple, y 13 de falso y verdadero. Cada tipo de preguntas se presentó con sus respectivas instrucciones a fin de facilitar la comprensión de su contenido y validar así la autenticidad de la prueba; se sometió a juicio de cinco personas expertas en el tema, y posteriormente se hizo la aplicación correspondiente a la muestra poblacional seleccionada.

Para obtener esta información se consideraron cinco etapas; en la primera se aplicó una prueba para reconocer la población seleccionada en el estudio; en la segunda, se aplicó un pretest con el fin de identificar el conocimiento que se tenía en relación con la enfermedad y el tipo de cuidado brindado a sus familiares enfermos de Alzheimer; en la tercera etapa, y con base en los resultados del anterior pretest, se planearon tres sesiones educativas personalizadas con el objeto de brindar información sobre los aspectos básicos de la fisiopatología de la enfermedad y de los cuidados integrales que debe recibir el paciente con EA en el hogar; en la cuarta etapa se llevó a cabo el desarrollo de las sesiones educativas con la familia de pacientes con EA, utilizando un tiempo aproximado de 40 a 60 minutos en cada sesión y, finalmente, en la quinta etapa, se aplicó el postest a las familias que participaron en las sesiones educativas.

Dichas entrevistas y sesiones educativas, se realizaron en el domicilio de cada familia, mediante citas previas concertadas telefónicamente.

\section{Resultados}

Para validar la hipótesis se tomó como base la prueba estadística de Mc Nemar, que valora el grado de significancia previa y posterior a una educación brindada, y que muestra el valor estadístico, $(>3,18)$, que es el valor de la distribución chi- cuadrado con un grado de libertad y $95 \%$ de confiabilidad (es decir que en 100 ensayos por lo menos en 95 se obtienen los mismos resultados); igualmente se muestre el valor de $p$, el cual debe ser $<0,05$ para rechazar la hipótesis nula o concluir que sí hubo efecto de las sesiones educativas.

Se mostró un notable mejoramiento de los conceptos de la familia relacionados con las medidas que deben tomarse y las actividades que propenden por la prevención de accidentes. En cuanto a medio ambiente y a recreación, se demostró en las preguntas 1 y 3 un efecto positivo significativo, después de la sesión de instrucción ( $p=0,03-0,00)$. En la primera pregunta en el postest $100 \%$ de los familiares contestaron correctamente contra 57\% del pretest. En cuanto a la pregunta 3, en el pretest $0,8 \%$ de los cuidadores contestó acertadamente y se logró fijar el conocimiento en cuanto al aspecto más importante de la prevención de accidentes después de la sesión educativa con un aumento hasta $100 \%$.

En las demás preguntas relacionadas con el medio ambiente, se observó un aumento positivo del nivel de conocimientos en la población en general. Al llevar a cabo la orientación, se hizo necesario hacer énfasis en la seguridad en el hogar, la adecuación del entorno, la conveniencia del cuidado de animales, la colaboración en las ocupaciones del hogar y la realización de actividades que tuvieran que ver con su antigua ocupación.

Sobre las precauciones que se deben tener a la hora del baño se encuentran falencias, pues aumentó el porcentaje de cuidadores que respondió incorrectamente en el postest; pasando de $42 \%$ a $68 \%$ después de la educación, lo que posiblemente se deba a que no se hizo mayor énfasis en el tema, sin aclarar los interrogantes que pudieran tener los cuidadores en este aspecto $\mathrm{y}$, además, por la falta de la ayuda didáctica que permitiera fijar el conocimiento de la familia.

La actitud del cuidador frente a los sentimientos y sexualidad del paciente se ve influenciada de forma positiva después de la realización de las sesiones educativas, observándose un nivel de significancia en la comunicación con el enfermo.

Respecto a la comunicación con el paciente se muestra un nivel de significancia de $(p=0,00)$, pues en el pretest $100 \%$ de los cuidadores contestaron incorrectamente; en 
cambio se observó efecto positivo de la sesión educativa con $65 \%$ de respuesta correctas (Figura 1).

Se evidencia que, luego de realizar la sesión, los cuidadores comprenden los cambios en la sexualidad del paciente de $40 \%$ pre a $65 \%$ postest $\mathrm{y}$, por ende, se mejora notablemente su concepto (100\% en el postest contra $65 \%$ en el pretest) en cuanto a cómo reaccionar frente a las diferentes conductas sexuales de su familiar.
Con los demás temas (visitas, serenidad, contacto con niños, reacción a conductas sexuales y cambios en la sexualidad) se logra establecer que aumenta el conocimiento de los cuidadores, sin considerarse significativa la prueba estadística; sin embargo, se logra el objetivo de la investigación con el incremento del conocimiento después de la educación impartida (Figura 2).

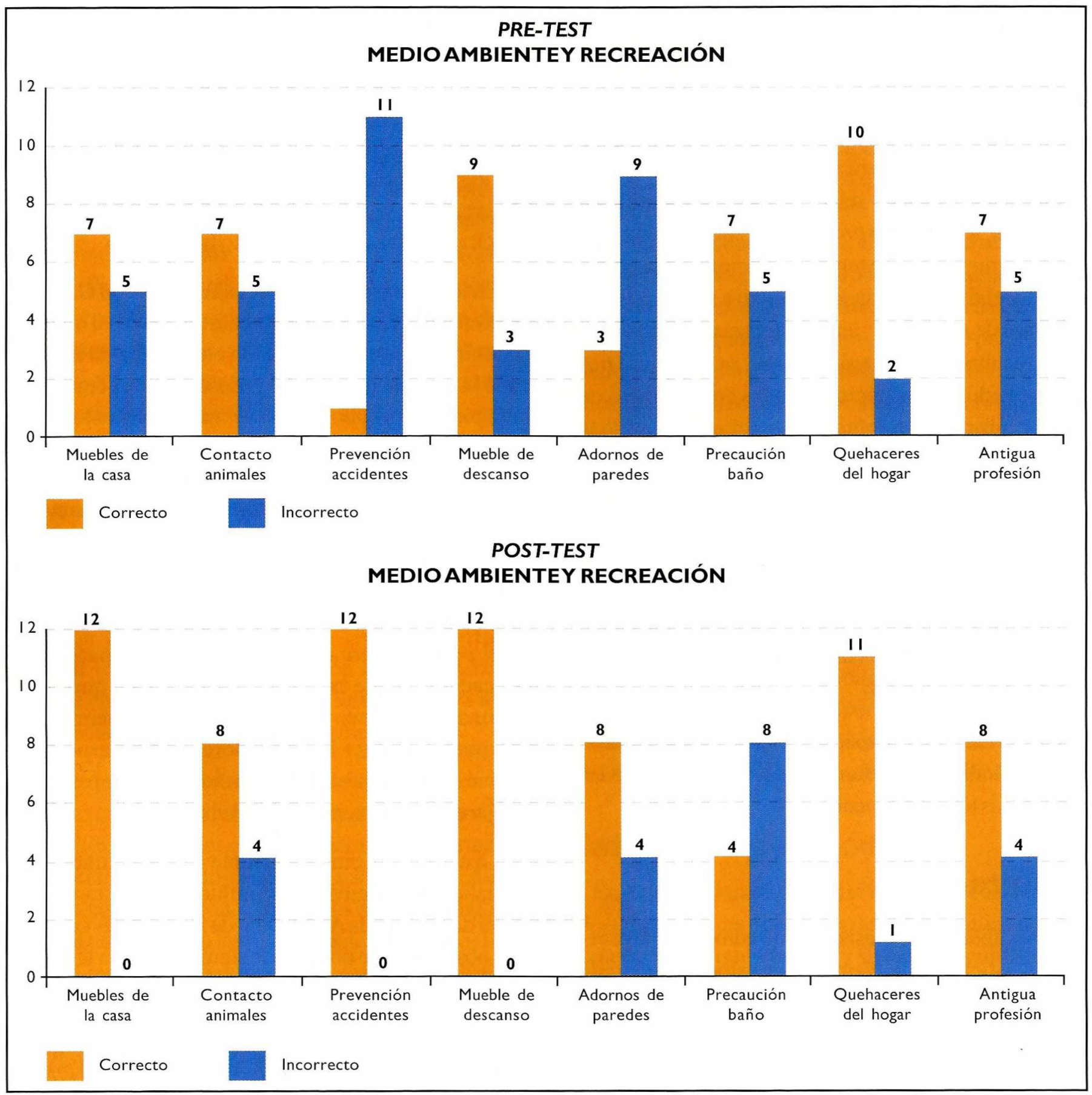

Figura I. Enfermedad de Alzheimer 
En relación con la nutrición como cuidado del paciente con EA, se observa, según el estudio, que los cuidadores conocen poco sobre la importancia de evitar las distracciones a la hora de comer, aspecto importante en toda persona, en especial del paciente con esta patología.

En la valoración del pretest, se encontró que $48 \%$ contestaron correctamente y se demostró un nivel de significancía positivo en el postest $(p=0,03)$ después de la sesión educativa, con $98 \%$ de respuestas correctas.

En cuanto a dar el tiempo prudente para que el paciente coma, se observa un cambio de $80 \%$ post contra $30 \%$ pretest. Esto permite reconocer cuales partes son importantes para reforzar las sesiones de instrucción. El postest demostró que los cuidadores comprendieron en su totalidad la importancia de dejar al paciente comer

\section{PRE-TEST}

\section{SENTIMIENTOS Y SEXUALIDAD}

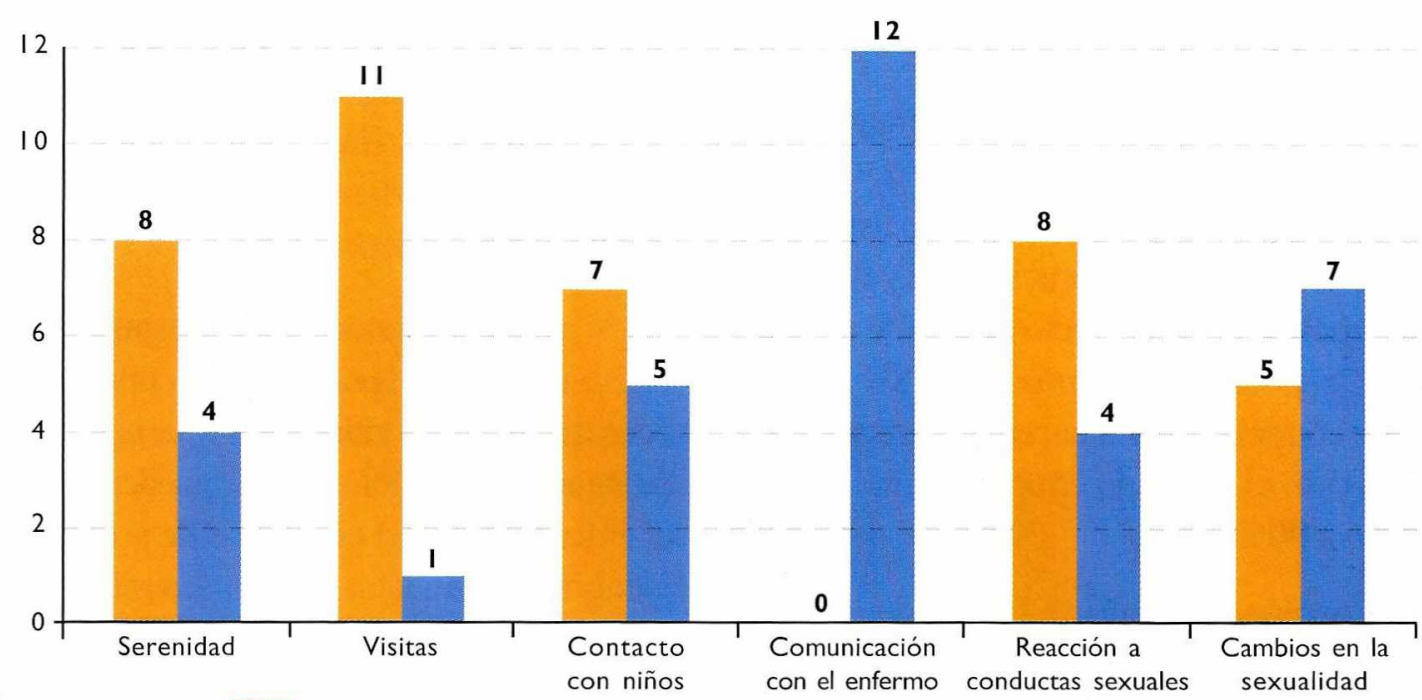

Correcto

Incorrecto

\section{POST-TEST SENTIMIENTOSY SEXUALIDAD}

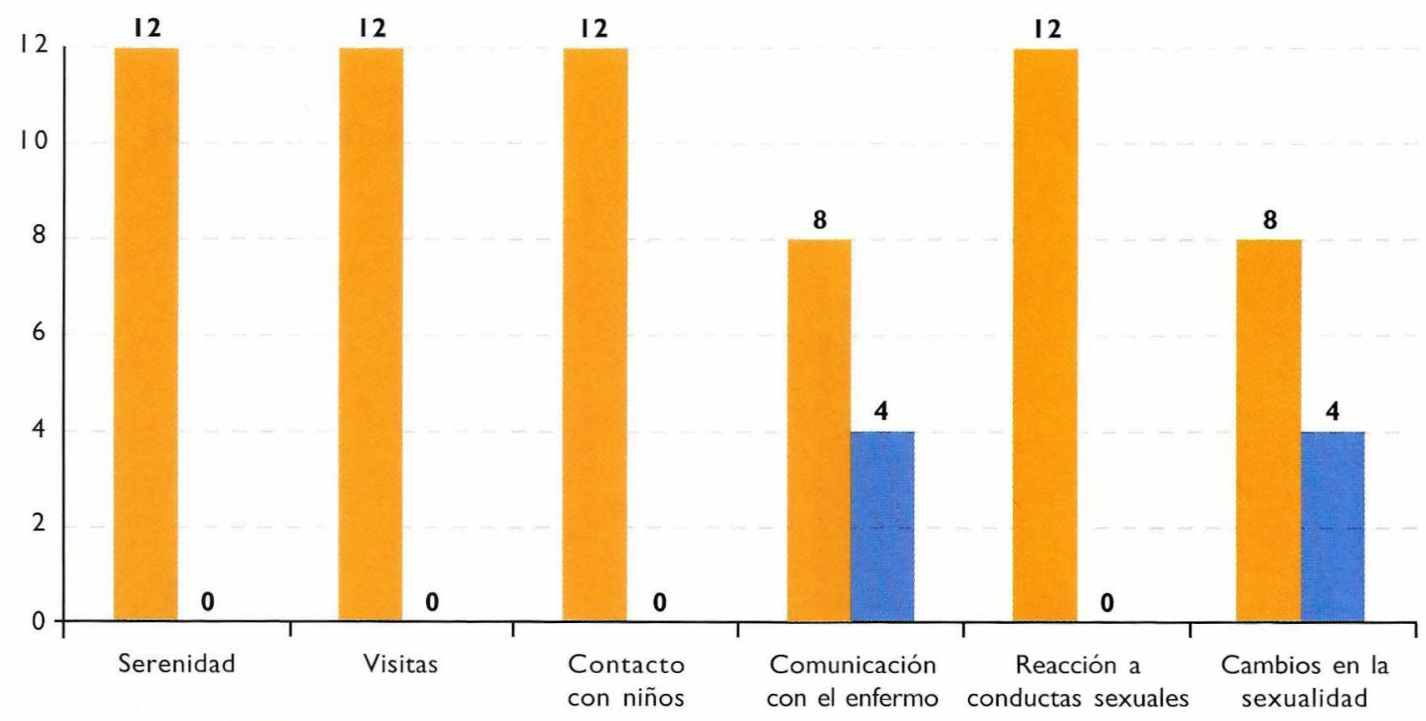

Correcto 
solo y a la vez observarlo, y cuando el paciente no desea comer, darle tiempo y esperar el momento en que desee hacerlo (Figura 3).

Se refleja el poco conocimiento sobre higiene que tienen los cuidadores. Según los datos obtenidos en el postest, $58 \%$ tienen los conceptos claros acerca de los cuidados específicos a la hora del cepillado, en comparación con $0 \%$ obtenido en el prestest ( $p=0,01)$; por esto se hace necesario reforzar durante la instrucción este aspecto. También hubo resultados significativos en las actividades más importantes antes del baño (100\% post, contra $58 \%$ pretest).

Los conocimientos de los cuidadores acerca de curaciones, oscilaron entre $0 \%$ y $100 \%$ postest. Ciento por ciento de los cuidadores tenían claro los aspectos más importantes al realizar una curación pre y postest, y $90 \%$ de cómo realizarse un correcto lavado de manos evidenciaron en el postest; mientras sólo 48\%, antes de la educación, conocían cómo hacer la correcta limpieza de una herida el nivel de conocimientos en el postest aumentó a $90 \%$

Con respecto al conocimiento del origen de las úlceras de decúbito, ya $58 \%$ de la población lo tenia claro y en el postest se complementó en la totalidad de las familias. Esto se debió posiblemente a que $42 \%$ de la población no tenía ningún conocimiento de lo que significaba una úlcera de decúbito, quizá por el diagnóstico reciente de la enfermedad de Alzheimer en su familiar que, en el momento del estudio, no presentaba ninguna lesión de ese tipo. Sin embargo, este aspecto, junto con el de incontinencia, fueron los de mayor impacto para el cuidador y muchos manifestaron no estar preparados para afrontar esta etapa respecto a sus familiares (Figura 4).

Se encontró una diferencia estadística significativa al comparar los resultados pre y postest en cuanto a la frecuencia con que se debe llevar el paciente al baño $(p=0,00)$ según la rutina impuesta por el cuidador (cada dos a cuatro horas) o cada vez que el paciente demuestre sentir deseos, (la primera opción es la correcta), contestada acertadamente por $100 \%$ de los cuidadores después de la educación.

Al evaluar el conocimiento después de la sesión educativa mediante el postest, se encontró, con respecto al concepto de la incontinencia urinaria (100\%), hubo $48 \%$ de signos y expresiones que se pueden identificar cuándo el enfermo tiene la necesidad de ir al baño y en $100 \%$ palabras que pueden usarse para referir la necesidad.

Los cuidadores tienen claro el concepto de incontinencia pero no el suficiente conocimiento sobre el manejo de la misma, por lo cual se debe enfatizar en este tema (Figura 5).

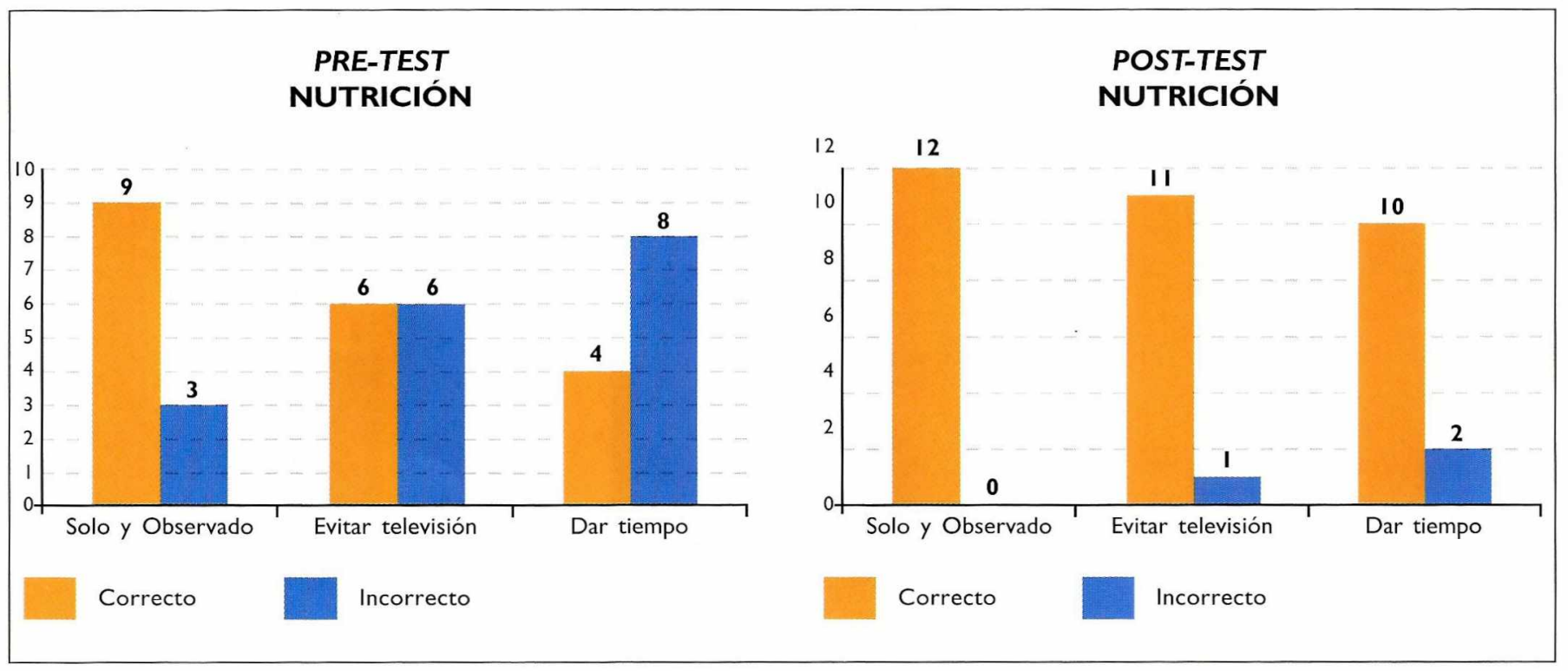

Figura 3. Enfermedad de Alzheimer 


\section{Discusión}

Durante la investigación se demostró la escasa intervención de enfermería en la educación a la familia del paciente con EA. Por lo tanto, las investigadoras vieron la necesidad de crear una pauta para brindar una orientación por medio de sesiones educativas personalizadas, dada la alta efectividad que se evidenció en el postest, lo cual demostró que esta orientación es adecuada y necesaria para la familia, ya que permite ofrecer el reconocimiento del paciente con EA como un ser biosicosocial con necesidades no sólo físicas, sino también emocionales y afectivas. Se crea así un mejor ambiente para él y su familia; además, hoy en día se acepta que lo más difícil de manejar en una persona demente es la alteración del comportamiento y la pérdida de independencia en su vida diaria. ${ }^{7}$

\section{PRE-TEST \\ HIGIENE, ÚLCERAS Y CURACIONES}

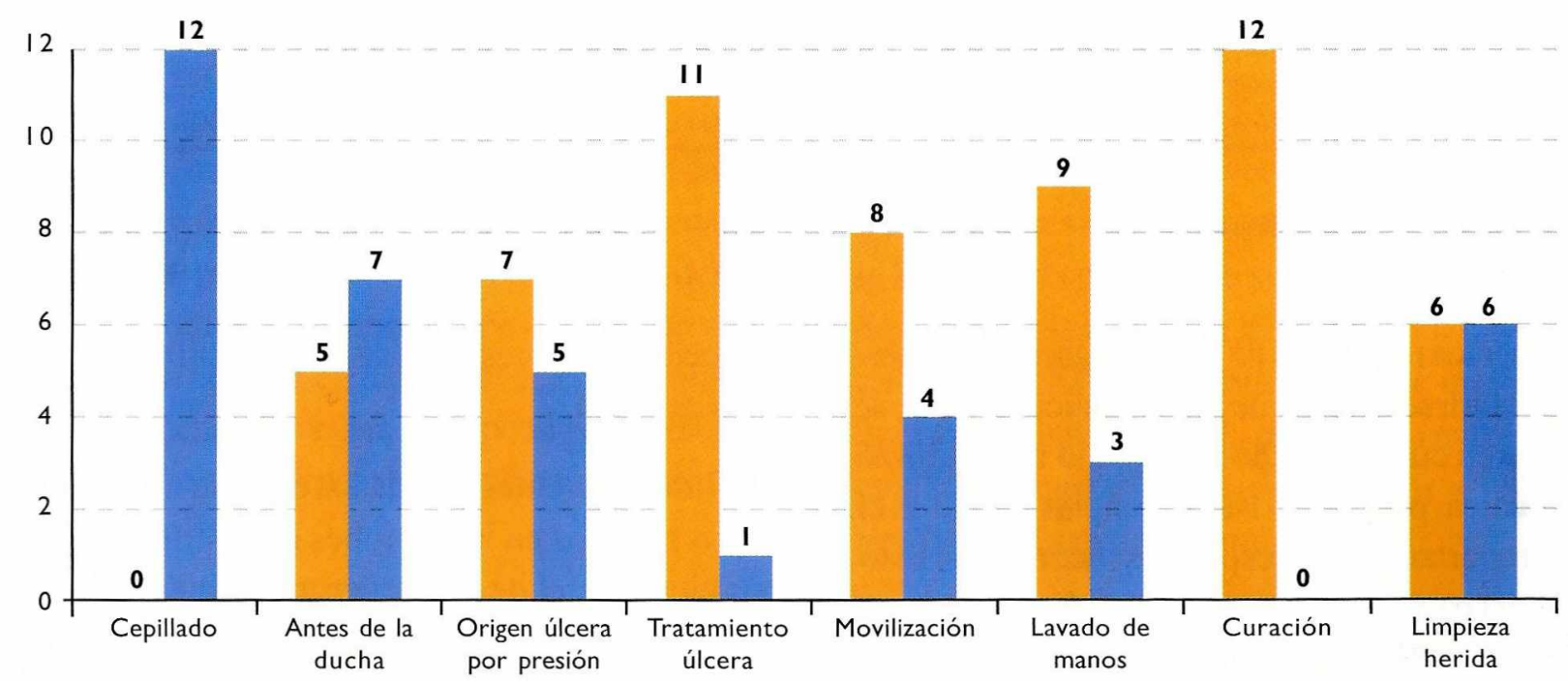

Correcto

Incorrecto

\section{POST-TEST \\ HIGIENE, ÚLCERAS Y CURACIONES}

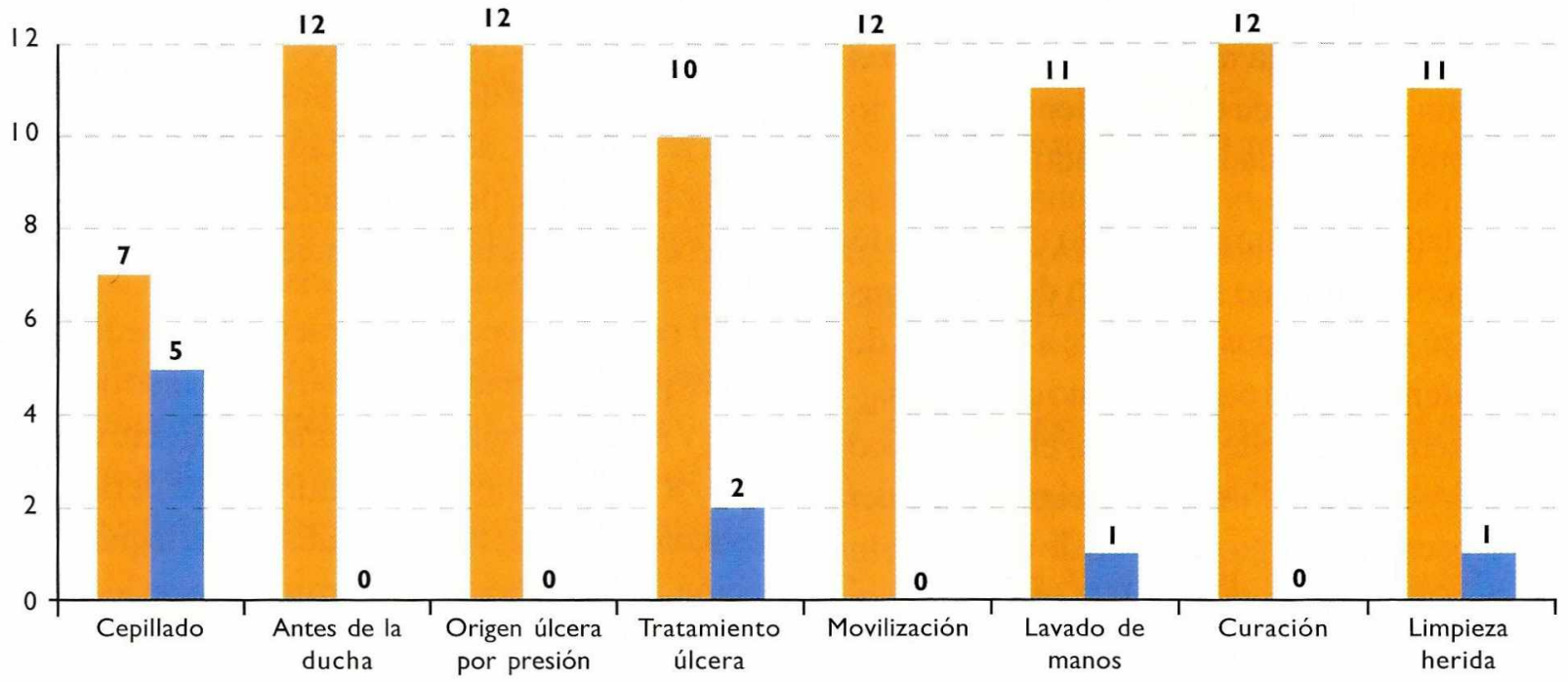

Correcto

Incorrecto

Figura 4. Enfermedad de Alzheimer 


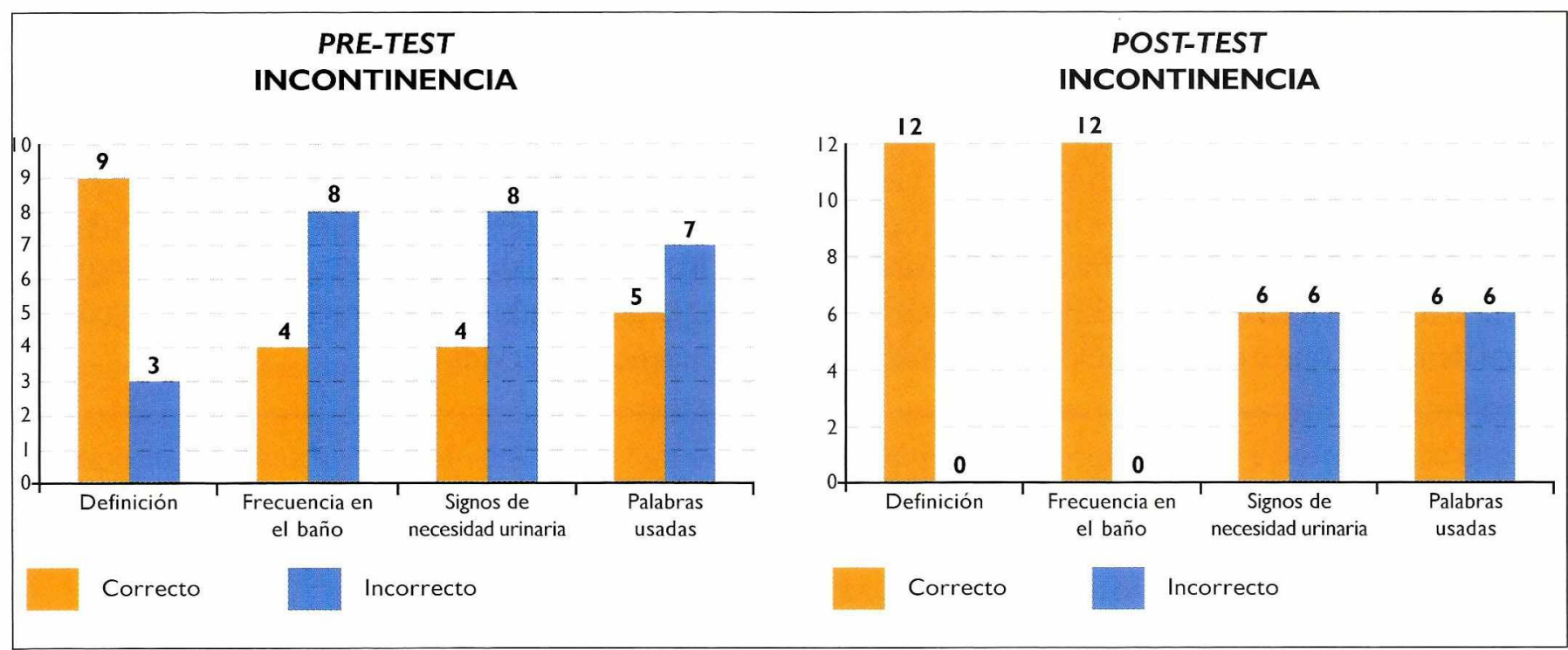

Figura 5. Enfermedad de Alzheimer

Al obtener los resultados de la investigación se observó que al referirse al tema de las precauciones que se deben tener en cuenta a la hora del baño no se utilizó ayuda didáctica, por esto existen falencias ya que en el postest se reportaron más respuestas incorrectas. Esto puede ser una pauta para que en un posible seguimiento de esta investigación se pueda efectuar un manejo adecuado, ya que las ayudas permiten fijar el conocimiento de una manera más acertada, lo cual es necesario para el bienestar tanto de la familia como del paciente con EA.

Acerca de los cambios de sexualidad en el paciente se observó notoriamente la mejora en las respuestas del postest, hubo un nivel de conocimiento mayor, lo que permite brindar un cuidado apropiado.

En la totalidad de las familias quedó claro todo lo relacionado al conocimiento del origen de las úlceras de decúbito; esto se debió posiblemente a que $42 \%$ de la población no tenia ningún conocimiento de lo que significaba una úlcera de decúbito, quizá por el diagnóstico reciente de la EA en su familiar que, en el momento del estudio, no presentaba ninguna lesión de ese tipo.Sin embargo, este aspecto junto con el de la incontinencia fueron los de mayor impacto para el cuidador, y muchos manifestaron no estar preparados para afrontar esta etapa en sus familiares. Es importante recordar que el paciente con EA requiere un cuidado especial, teniendo en cuenta que necesita un análisis múltiple ya que cada caso es único. ${ }^{8}$

Hubo diferentes resultados de cada uno de los cuidados tratados en las sesiones de instrucción, como, por ejemplo, el de mantener un ambiente adecuado modificando algunas cosas en la organización y distribución de su hogar, muebles de la casa, adornos de paredes, precauciones en el baño; esto también para prevenir los accidentes.

A la vez es indispensable no olvidar la parte de la recreación, los sentimientos (de familiares y paciente), y la sexualidad, ya que todos estos aspectos toman fuerza en el área mental, además están muy interferidos en la patología y no se pueden abandonar creyendo que no es necesario tratarlos en el paciente.

El paciente necesita distraerse por medio de diferentes juegos recreativos para mantenerse ocupado en algo y seguir trabajando su parte cognoscitiva, aunque poco a poco se vaya perdiendo, no se deben apartar los sentimientos de toda la familia ya que lograr una aceptación de la enfermedad permite que se le brinde un cuidado lleno de amor, comprensión y respeto, lo cual es prioritario puesto que a pesar de que lo sientan alejado y a veces fuera de contexto, no deja de percibir cómo lo está tratando su familia. 
Hay que manejar el área física del paciente, procurando llevar una buena técnica de alimentación y una adecuada dieta para su edad. Estas personas con Alzheimer requieren cuidados como los de cualquier otra persona (baño, actividades físicas, mentales, alimentación, recreación, sexualidad, etc), la diferencia está en la forma de brindarlos, también en la manera de abordar al paciente $y$, además, recordando que sus facultades mentales se están perdiendo mucho más rápido que en otros ancianos.

En una etapa más avanzada, se pueden presentar problemas de incontinencia, úlceras, dificultades para la marcha, incapacidades de realizar su aseo personal, $\mathrm{y}$, por últimas, necesitar un cuidado estricto en cama, debido que al pasar el tiempo se pueden ir presentando más problemas por la degeneración progresiva del paciente. En las sesiones de instrucción se manejaron aspectos como la higiene, el manejo de úlceras y curaciones, la incontinencia y mecánica corporal para movilizar adecuadamente al paciente sin perjudicar la salud de la persona que lo haga.

Todos los temas tratados llevan un cuidado de enfermería ya que se hace necesaria esta intervención para mejorar la calidad de vida de estos pacientes y sus familias.

En el desarrollo de la investigación se logró valorar que en el campo de la salud mental el profesional de enfermería no manifiesta el interés que demuestra por otras áreas, siendo una de las áreas que permite difundir el ejercicio profesional hacia una parte esencial del ser humano, lo que motivó a las investigadoras a intervenir en este nuevo campo, ya que se hace necesaria la presencia de la enfermera en la educación a este nivel.

Esta investigación no solo aportó algunos conocimientos a las familias sino que también clarifico y verificó conceptos previos que muy seguramente ayudarán a evitar maltratos por desconocimiento o falta de información.

\section{Conclusiones}

Dados los resultados encontrados en el pretest se puede concluir que es necesario dar orientación a los cuidadores de los pacientes con EA sobre el cuidado integral que deben recibir en el hogar, para mejorar su calidad de vida.

También se pudo observar en cuáles temas se necesitó hacer más énfasis (precauciones a la hora del baño, incontinencia, ulceras de decúbito, higiene oral, limpieza de heridas, comunicación con el enfermo, prevención de accidentes, adaptación del entorno) para así reforzar y afianzar los conocimientos acerca de los cuidados en el hogar, los cuales fueron objetivos planteados en el proyecto de investigación.

En términos generales se mostró que las sesiones de instrucción sí surtieron efecto en los conocimientos de los cuidadores respecto al manejo de los pacientes con la EA, pues la prueba fue significativa en los casos en donde el pretest evidenció poco conocimiento en los cuidadores. Es importante decir que en los casos en que no fue significativa la prueba, era evidente que los cuidadores conocían ya los cuidados y, entonces, no tenía porqué existir un cambio.

Gracias a la experiencia que se obtuvo durante la realización del proyecto se ha logrado ver que la población del estudio estuvo atenta a cada una de las sesiones de instrucción. Se puede concluir que estas sesiones personalizadas hacen que la orientación sea más directa $\mathrm{y}$ formal teniendo en cuenta que cada persona tiene una forma diferente de captar y procesar la información.

Se debe recordar que esta es una patología del nuevo milenio, donde se ha empezado a analizar profundamente en todos sus aspectos ${ }^{9}$ para crear así nuevas estrategias de cuidado.

\section{Lecturas recomendadas}

- Adams Víctor. R. Manual de Principios de Neurología. $6^{a}$ ed. Bogotá: Mc Graw Hill; 411.

- Arboleda H, Arboleda G H, Yunis J J, Pardo R, Gómez C.M. Benavides J. Grupo de trabajo interdisciplinario en Demencia, Unidad de Neurología- Instituto de Genética, Universidad Nacional de Colombia.

- Asociación Nacional de Enfermeras Seccional Cundinamarca 266/ 1996 Enero 25. Capitulo V. Art. 13.pg. 13 
- Castañeda M, Villegas LCA, Arias R LF, Vieco B, Sepúlveda Falla Da, Bedoya G, Lopera F. Medellín Grupo de Neurociencias de Antioquia. Reunión Latinoamericana de Alzheimer 3a: 28-31, Cuba mar 2000. Lluch R, Cuesta, Marages. El cuidador principal en la enfermedad de Alzheimer. Mislata, Valencia: A D A M, 2001. En www.valencia.arg. Montañes P, Matallana D, Cano C, Plata S, García R, Gil F.
- Clínica de Memoria, Epidemiología clínica y Bioestadística. Bogotá. Universidad Javeriana Departamento de Psicología.

- Orozco M, Arango G, Pardo R, Grupo de trabajo interdisciplinario de demencia,

- Unidad de Neurología. Instituto de Genética, Universidad Nacional de Colombia.

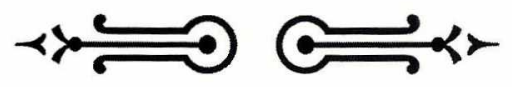

\section{FUNDACIÓN UNIVERSITARIA DE CIENCIAS DE LA SALUD HOSPITAL DE SAN JOSE}

Personería Jurídica No. 10917 del 01-12-1976 Resolución Ministerio de Educación Nacional No. 0125

Facultad de Instrumentación Quirúrgica

Código ICFES 270246100281100111100

Título otorgado

Instrumentador Quirúrgico
Oficina de admisiones: Calle 10 No. $18-75$ Edificio docente, $7^{\text {er }}$ piso PBX. 5998977 • 5998842 TELEFAX 2019867 • 2018938

E-mail: instrumentacion@fucsalud.edu.co

hppt: www.fucsalud.edu.co

F.

I SEMESTRE

- Biología

- Morfofisiologíal

- Física

- Química

- Introducción a la

instrumentación

- Comunicación

oral y escrita

- Informática

\begin{tabular}{l|ll|}
\hline II SEMESTRE & III SEMESTRE & IV SEMESTRE \\
- Microbiología & - Patología I & - Patología II \\
- Ecología & - Cuidados básicos & - Epidemiología \\
- Morfofisiología II & en salud & - Técnicas \\
- Esterilización & - Estadística & quirúrgicas III \\
- Bioquímica & - Farmacología & - Técnicas de \\
- Técnicas & - Anestesia & instrumentación II \\
quirúrgicas I & - Técnicas & - Inglés II \\
- Técnicas de & quirúrgicas II & - Constitucion \\
instrumentación & - Técnicas de & política \\
- Sociología & instrumentacion II & yderecho en salud \\
& - Inglés I & - Ética general \\
& - Psicología & - Administración \\
& & general \\
& & -Investigación I
\end{tabular}

V SEMESTRE

- Práctical

- Bioética

- Administración

hospitalaria

- Investigación II

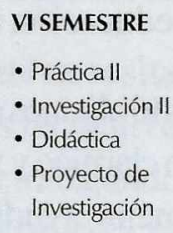

VII SEMESTRE

- Práctica III

- Proyecto de

Investigación

- Mercadeo

- Seminario de Investigación
VIII SEMESTRE

- Práctica IV 
- Castañeda M, Villegas LCA, Arias R LF, Vieco B, Sepúlveda Falla Da, Bedoya G, Lopera F. Medellín Grupo de Neurociencias de Antioquia. Reunión Latinoamericana de Alzheimer $3^{\text {a: }}$ :28-31, Cuba mar 2000. Lluch R, Cuesta, Marages. El cuidador principal en la enfermedad de Alzheimer. Mislata, Valencia: A D A M, 2001. En www.valencia.arg. Montañes P, Matallana D, Cano C, Plata S, García R, Gil F.
- Clínica de Memoria, Epidemiología clínica y Bioestadística. Bogotá. Universidad Javeriana Departamento de Psicología.

- Orozco M, Arango G, Pardo R, Grupo de trabajo interdisciplinario de demencia,

- Unidad de Neurología. Instituto de Genética, Universidad Nacional de Colombia.

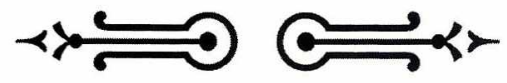

\section{FUNDACIÓN UNIVERSITARIA DE CIENCIAS DE LA SALUD HOSPITAL DE SAN JOSE}

Facultad de Instrumentación Quirúrgica

Código ICFES 270246100281100111100

Título otorgado

\section{Instrumentador Quirúrgico}

Oficina de admisiones: Calle 10 No. $18-75$ Edificio docente, $1^{\text {er }}$ piso PBX. 5998977 • 5998842 TELEFAX 2019867 • 2018938

E-mail: instrumentacion@fucsalud.edu.co

hppt: www.fucsalud.edu.co

\section{(s)}

I SEMESTRE

- Biología

- Morfofisiología I

- Física

- Química

- Introducción a la

instrumentación

- Comunicación

oral y escrita

- Informática

\section{SEMESTRE}

- Microbiología

- Ecología

- Morfofisiología II

- Esterilización

- Bioquímica

- Técnicas

quirúrgicas |

- Técnicas de

instrumentación

- Sociología

III SEMESTRE
- Patología I
- Cuidados básicos
en salud
- Estadística
- Farmacología
- Anestesia
- Técnicas
quirúrgicas II
- Técnicas de
instrumentacion II
- Inglés I
- Psicología

- Psicología
IV SEMESTRE

- Patología II

- Epidemiología

- Técnicas

quirúrgicas III

- Técnicas de

instrumentación II

- Inglés II

- Constitucion

política

y derecho en salud

- Ética general

- Administración

general

- Investigación I

\section{SEMESTRE}

- Práctical

- Bioética

- Administración

hospitalaria

- Investigación II

\section{SEMESTRE}

- Prácticall

- Investigación I

- Didáctica

- Proyecto de

Investigación
VII SEMESTRE

- Práctica III

- Proyecto de

Investigación

- Mercadeo

- Seminario de Investigación
VIII SEMESTRE

- Práctica IV 